\title{
Pneumonia induced by eribulin mesylate in a patient with recurrent breast cancer
}

\author{
Hiroo Ishida $\cdot$ Tetsuya Homma $\cdot$ Koko Ishida $\cdot$ Tomohide Sugiyama $\cdot$ \\ Sojiro Kusumoto • Takao Shirai • Masanao Nakashima - Tsukasa Ohnishi • \\ Takashi Hirose
}

Received: 30 August 2012/Accepted: 19 November 2012/Published online: 20 December 2012

(C) The Japan Society of Clinical Oncology 2012

\begin{abstract}
A 48-year-old woman with breast cancer overexpressing human epidermal growth factor receptor type 2 was given eribulin mesylate, which induced pneumonia. Drug-induced pneumonia related to eribulin mesylate was diagnosed on the basis of clinical and radiographic findings and a positive reaction in the druginduced lymphocyte stimulating test. The symptoms and radiographic findings improved without corticosteroid therapy. Careful attention should be paid to the possible signs and symptoms of lung injury in patients who receive eribulin mesylate or other chemotherapeutic agents.
\end{abstract}

Keywords Drug-induced pneumonia .

Antineoplastic drug $\cdot$ Eribulin

\section{Introduction}

Eribulin mesylate is a synthetic analogue of halichondrin $\mathrm{B}$, which is isolated from the marine sponge Halichondria okadai [1]. The binding of eribulin mesylate to tubulin inhibits the growth phase of microtubules. This interference with microtubular function leads to blockade of the G2-M phase of the cell cycle, disruption of mitotic spindles, and apoptosis of tumor cells [1, 2]. In phase II studies, breast cancers resistant to anthracycline and taxane showed responses to eribulin mesylate [3, 4]. Subsequently,

H. Ishida $(\bowtie) \cdot$ T. Homma $\cdot$ K. Ishida $\cdot$ T. Sugiyama $\cdot$

S. Kusumoto - T. Shirai · M. Nakashima - T. Ohnishi ·

T. Hirose

Division of Respiratory Medicine and Allergology,

Department of Internal Medicine, Showa University

School of Medicine, 1-5-8 Hatanodai, Shinagawa,

Tokyo 142-8666, Japan

e-mail: hishida@med.showa-u.ac.jp eribulin mesylate was approved by the Ministry of Health, Labour and Welfare in Japan because it has improved survival in patients with metastatic breast cancer who had received multiple previous treatments [5].

In a phase I study of eribulin mesylate, the most common dose-limiting toxicities were neutropenia, febrile neutropenia, and fatigue $[6,7]$. Although it has been reported that the incidence of eribulin mesylate-induced pneumonia was $1.2 \%$ in a Japanese phase II study [8], the detailed clinical course with pneumonia induced by eribulin mesylate has not been previously reported. Therefore, we now report a case of eribulin mesylate-induced pneumonia.

\section{Case report}

In March 2006, a 48-year-old woman was found to have breast cancer with a pathological diagnosis of invasive ductal carcinoma and a clinical stage of T2N1aM0, stage IIB. Immunohistochemical staining was negative for estrogen receptor and positive for progesterone receptor and overexpressed (3+) human epidermal growth factor receptor type 2 (HER2). Neoadjuvant chemotherapy with 5-fluorouracil, epirubicin, and cyclophosphamide followed by weekly paclitaxel was performed, and right mastectomy and right axillary lymph node resection was then performed. After surgery, a luteinizing hormone-releasing hormone agonist and tamoxifen were administered as adjuvant therapy.

Recurrent disease was detected at the right chest wall and right axillary lymph nodes during administration of tamoxifen in January 2011. Therefore, the patient was given the combination chemotherapy of vinorelbine plus trastuzumab. Because the patient failed to respond to this treatment, she was given the combination chemotherapy of capecitabine 
plus trastuzumab and the combination chemotherapy of gemcitabine plus trastuzumab. However, disease continued to progress, and the combination chemotherapy of eribulin $\left(1.4 \mathrm{mg} / \mathrm{m}^{2}\right.$ on day 1 and 8 every 21 days) plus trastuzumab ( $2 \mathrm{mg} / \mathrm{kg}$ once a week) was started on 4 February 2012. The next day, fluctuating fever and cough developed. Although her physician prescribed levofloxacin and acetaminophen, the symptoms did not improve. Therefore, she was referred to our institution on 10 February 2012, because of a fever of $38^{\circ} \mathrm{C}$ and cough lasting 1 week.

She had no history of smoking and no history of lung disease. She had taken loxoprofen and pregabalin daily for chest pain. She had cough and mild fatigue (performance status of 1) on admission to our institution. Physical examination showed a blood pressure of $96 / 56 \mathrm{mmHg}$, a pulse rate of 82 beats per minute, a temperature of $39.5^{\circ} \mathrm{C}$, a respiratory rate of 12 breaths per minute, and an oxygen saturation of $96 \%$ while breathing room air. There were no abnormal lung sounds. The remainder of the general physical examination showed no abnormalities.

Hematological studies showed leukopenia $\left(2,400 / \mathrm{mm}^{3}\right)$ and thrombocytopenia $\left(103,000 / \mathrm{mm}^{3}\right)$ that might be myelosuppression induced by eribulin mesylate. The differential white blood count was neutrophils $50.2 \%$, lymphocytes $45.2 \%$, monocytes $2.9 \%$, and eosinophils $0.1 \%$. Biochemical tests revealed high levels of lactate dehydrogenase (222 IU/L) and C-reactive protein $(1.41 \mathrm{mg} / \mathrm{dL})$. However, levels of KL-6 (206.0 U/mL), surfactant protein-A $(27.7 \mathrm{ng} / \mathrm{mL})$, and surfactant proteinD $(17.3 \mathrm{ng} / \mathrm{mL})$ were within their normal ranges. Serum antibodies against Mycoplasma pneumoniae, Chlamydophila pneumoniae, or Cytomegalovirus were not found. Plasma level of 1,3-beta-D-glucan $(4.0 \mathrm{ng} / \mathrm{mL})$ was within normal range. The rapid antigen test for Influenza virus $A$ and $B$ were negative. The rapid urinary antigen test for Legionella pneumophila and Streptococcus pneumoniae were negative. Sputum culture yielded no pathogenic bacteria. Pulmonary function testing showed normal ventilatory function (percent vital capacity, $92.4 \%$; forced expiratory volume in $1 \mathrm{~s}$ as a percentage of forced vital capacity, $75.5 \%$ ).

Chest X-ray films showed a ground-glass shadow in the right lower lung (Fig. 1a), and computed tomography (CT) of the chest revealed extensive ground-glass opacities (GGOs) independent of the bronchial distribution in both lungs (Fig. 1b). High-resolution CT (HRCT) of the chest showed focal GGO and septal thickening (Fig. 1c). Because CT findings suggested noninfectious interstitial pneumonia, the patient was admitted to our hospital, and eribulin mesylate was discontinued on February 10, 2012.

The drug-induced lymphocyte stimulating test (DLST) was performed with a sample of the patient's blood obtained before eribulin mesylate was discontinued. The
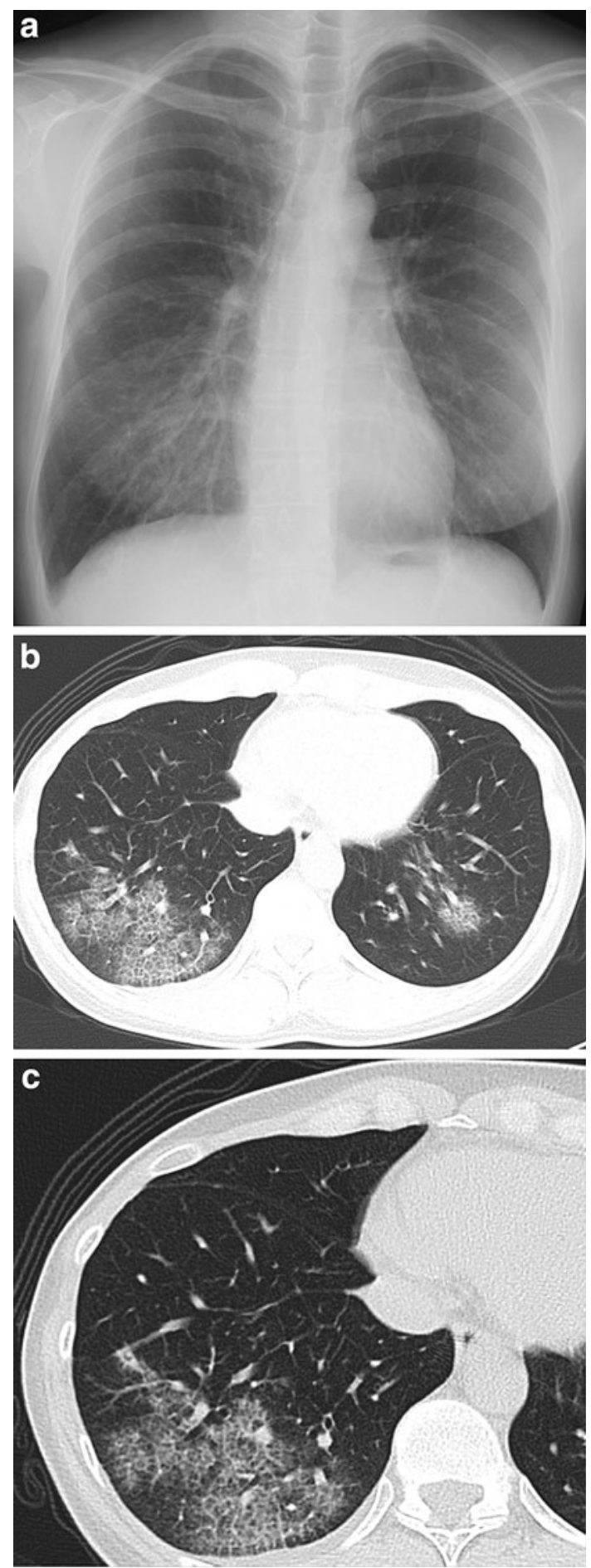

Fig. 1 Chest X-ray films show a ground glass shadow in the right lower lung (a), and computed tomography of the chest shows bilateral ground glass opacity in the dorsal lower lung (b), and high-resolution computed tomography of the chest shows focal ground glass shadow and septal thickening (c), at the time of admission

measured value was 2,356 counts per minute (cpm), compared with $124 \mathrm{cpm}$ for the control (stimulation index, 19.0). This result showed a positive reaction. Concurrent 

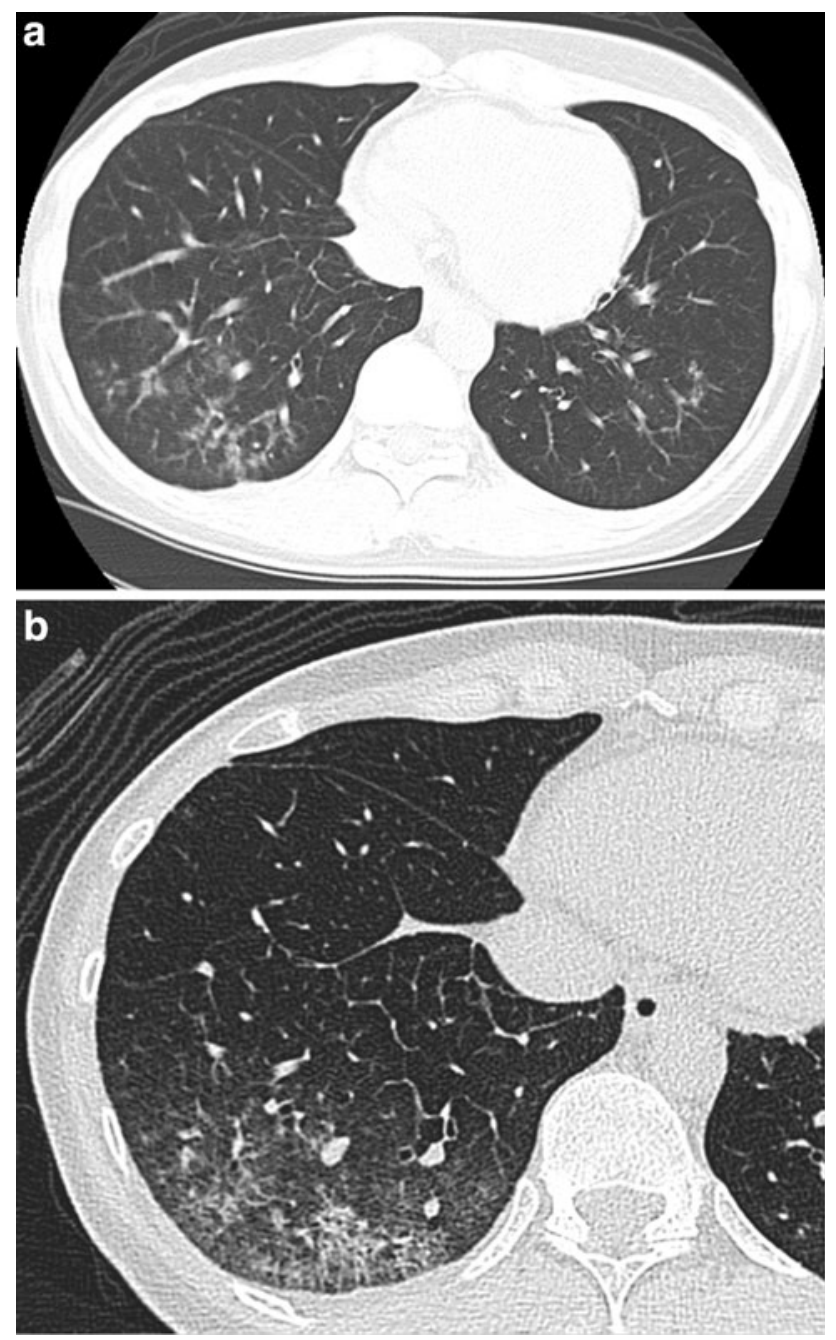

Fig. 2 Computed tomography (a) and high-resolution computed tomography (b) on the 11th hospital day shows the disappearance of ground glass opacity

used drugs such as trastuzumab, loxoprofen, and pregabalin showed a negative reaction with DLST.

Though bronchoalveolar lavage and transbronchial lung biopsy were considered, the fever resolved on the third hospital day. Furthermore, the ground glass shadow on chest X-ray films showed improvement. Therefore, we carefully observed the patient's condition. Chest CT on the 11th hospital day showed that the GGO had resolved (Fig. 2a, b), and the patient was discharged. Although trastuzumab monotherapy was administered after discharge, GGO has not been found on chest CT.

\section{Discussion}

To our knowledge, this is the first detailed report of the clinical course with pneumonia induced by eribulin mesylate. Drug-induced pneumonia is a severe adverse event during cancer chemotherapy. It has been reported that $1 \%$ of patients who received cytotoxic anticancer agents developed drug-induced pneumonia [9]. Importantly, all the anticancer agents have the potential to induce lung injury.

However, the criteria and methods for diagnosis of druginduced pneumonia have not been established. A history of drug exposure is the most important criterion for the diagnosis of drug-induced pneumonia. Additionally, other causes of lung injury, e.g., infection, fluid overload, pulmonary edema, and metastasis from the underlying neoplasm, must be excluded [10]. In the diagnosis of interstitial pneumonia, pathological examination of lung tissues obtained at thoracotomy or video-associated thoracic surgery is recommended. However, specimens from transbronchial lung biopsy are not adequate for diagnosing interstitial pneumonia because the volume of obtained tissue is too small.

Fortunately, new diagnostic imaging methods, such as HRCT, can help distinguish infectious lung disease from noninfectious disease, such as drug-induced pneumonia. In general, CT shows the consolidation or GGO that spreads along bronchial distribution in infectious pneumonia. On the other hand, the predominant CT finding of noninfectious pneumonia is diffuse multifocal GGO that spreads independently of the bronchial distribution [11]. In the present case, CT revealed GGO that spread independently of the bronchial distribution, a finding that suggested a noninfectious lung disease. Furthermore, bilateral focal GGO which included septal thickening suggested druginduced pneumonia of organizing pneumonia-like pattern [12].

Although the DLST has a sensitivity for drug-induced pneumonia of only $70 \%$ and has yielded false positive results in healthy volunteers, it remains useful for the diagnosis of drug-induced pneumonia [13]. Drug-induced pneumonia should be diagnosed on the basis of physical, radiologic, pathological, and serological findings, such as those of the DLST. In the present case, CT findings, the positive result of the DLST, and the symptomatic and radiological improvement without corticosteroid or antibiotics strongly suggested that the pneumonia had been induced by eribulin mesylate. Although the efficacy and safety of eribulin plus trastuzumab has not been adequately evaluated, it has been reported that the continuation of trastuzumab prolonged survival in patients with HER2positive, advanced breast cancer after progression with prior trastuzumab-containing chemotherapy [14]. Therefore, eribulin plus trastuzumab were concurrently administered after the patient provided written informed consent.

Although no standard therapy for drug-induced pneumonia has been established, corticosteroids have generally 
been administered. In particular, corticosteroids should be administered immediately if pneumonia is induced by docetaxel or gemcitabine, because these agents often cause severe lung injury, such as diffuse alveolar damage [11]. On the other hand, paclitaxel has been reported to induce a transient pulmonary infiltration $48 \mathrm{~h}$ after administration which improves over several days without corticosteroids. Paclitaxel-induced pneumonia has been considered a type of hypersensitivity reaction [15]. Similarly, eribulin mesylate might also cause pneumonia through a hypersensitivity reaction, because our patient began to have fluctuating fever and cough the day after she first received eribulin mesylate and because both symptoms and radiographic findings improved promptly without corticosteroid therapy after eribulin mesylate had been discontinued.

In conclusion, careful attention should be paid to the possible signs and symptoms of lung injury in patients who receive eribulin mesylate or other chemotherapeutic agents.

Conflict of interest No author has any conflict of interest.

\section{References}

1. Jordan MA, Kamath K, Manna T et al (2005) The primary antimitotic mechanism of action of the synthetic halichondrin E7389 is suppression of microtubule growth. Mol Cancer Ther 4:1086-1095

2. Smith JA, Wilson L, Azarenko O et al (2010) Eribulin binds at microtubule ends to a single site on tubulin to suppress dynamic instability. Biochemistry 49:1331-1337

3. Vahdat LT, Pruitt B, Fabian CJ et al (2009) Phase II study of eribulin mesylate, a halichondrin B analog, in patients with metastatic breast cancer previously treated with an anthracycline and a taxane. J Clin Oncol 27:2954-2961
4. Cortes J, Vahdat L, Blum JL et al (2010) Phase II study of the halichondrin $B$ analog eribulin mesylate in patients with locally advanced or metastatic breast cancer previously treated with an anthracycline, a taxane, and capecitabine. J Clin Oncol 28:3922-3928

5. Cortes J, O'Shaughnessy J, Loesch D et al (2011) Eribulin monotherapy versus treatment of physician's choice in patients with metastatic breast cancer (EMBRACE): a phase 3 open-label randomised study. Lancet 377:914-923

6. Goel S, Mita AC, Mita M et al (2009) A phase I study of eribulin mesylate (E7389), a mechanistically novel inhibitor of microtubule dynamics, in patients with advanced solid malignancies. Clin Cancer Res 15:4207-4212

7. Tan AR, Rubin EH, Walton DC et al (2009) Phase I study of eribulin mesylate administered once every 21 days in patients with advanced solid tumors. Clin Cancer Res 15:4213-4219

8. Aogi K, Iwata H, Masuda N et al (2012) A phase II study of eribulin in Japanese patients with heavily pretreated metastatic breast cancer. Ann Oncol 23:1441-1448

9. Dimopoulou I, Bamias A, Lyberopoulos P et al (2006) Pulmonary toxicity from novel antineoplastic agents. Ann Oncol 17:372-379

10. Camus P, Fanton A, Bonniaud P et al (2004) Interstitial lung disease induced by drugs and radiation. Respiration 71:301-326

11. Muller NL, White DA, Jiang $\mathrm{H}$ et al (2004) Diagnosis and management of drug-associated interstitial lung disease. Br J Cancer 91(Suppl 2):S24-S30

12. Rossi SE, Erasmus JJ, McAdams HP et al (2000) Pulmonary drug toxicity: radiologic and pathologic manifestations. Radiographics 20:1245-1259

13. Hirata S, Hattori N, Kumagai K et al (2009) Lymphocyte transformation test is not helpful for the diagnosis of methotrexateinduced pneumonitis in patients with rheumatoid arthritis. Clin Chim Acta 407:25-29

14. von Minckwitz G, Schwedler K, Schmidt M et al (2011) Trastuzumab beyond progression: overall survival analysis of the GBG 26/BIG 3-05 phase III study in HER2-positive breast cancer. Eur J Cancer 47:2273-2281

15. Ramanathan RK, Belani CP (1996) Transient pulmonary infiltrates: a hypersensitivity reaction to paclitaxel. Ann Intern Med $124: 278$ 